\title{
Bifurcation from infinity for reaction-diffusion equations under nonlinear boundary conditions
}

\author{
Nsoki Mavinga \\ Department of Mathematics and Statistics, Swarthmore College, \\ Swarthmore, PA 19081-1390, USA (mavinga@swarthmore.edu) \\ Rosa Pardo \\ Departamento de Matemática Aplicada, Universidad Complutense \\ de Madrid, 28040 Madrid, Spain (rpardo@mat.ucm.es)
}

(MS received 2 March 2015; accepted 17 December 2015)

\begin{abstract}
We consider reaction-diffusion equations under nonlinear boundary conditions where the nonlinearities are asymptotically linear at infinity and depend on a parameter. We prove that, as the parameter crosses some critical values, a resonance-type phenomenon provides solutions that bifurcate from infinity. We characterize the bifurcated branches when they are sub- or supercritical. We obtain both Landesman-Lazer-type conditions that guarantee the existence of solutions in the resonant case and an anti-maximum principle.

Keywords: Steklov eigenvalues; elliptic equations; nonlinear boundary conditions; bifurcation

2010 Mathematics subject classification: Primary 35j66
\end{abstract}

\section{Introduction}

Consider the following nonlinear elliptic problem with nonlinear boundary conditions:

$$
\left.\begin{array}{rlrl}
-\Delta u+u & =\lambda m(x) u+f(\lambda, x, u) & & \text { in } \Omega, \\
\frac{\partial u}{\partial \nu} & =\lambda \rho(x) u+g(\lambda, x, u) & & \text { on } \partial \Omega,
\end{array}\right\}
$$

where $\Omega$ is a bounded domain in $\mathbb{R}^{N}(N \geqslant 2)$ with smooth boundary, $\partial / \partial \nu:=\nu \cdot \nabla$ is the (unit) outer normal derivative, the parameter $\lambda \in \mathbb{R}$, the weight functions $m, \rho \geqslant 0,(m, \rho) \neq(0,0)$ and the functions $f$ and $g$ are sublinear at infinity.

Problems with nonlinear boundary conditions have recently attracted considerable attention. In this paper we analyse the bifurcation induced by nonlinearities in both the differential equation and the boundary condition. The existence of solutions of nonlinear problems when the nonlinearity is in the differential equation and the boundary condition is Dirichlet has been widely studied using (among other techniques) bifurcation theory (see, for example, [4]). However, problems with nonlinear boundary conditions have been less widely studied. For the Laplace operator with nonlinear boundary conditions see, for example, $[5-7,9,17,19]$ and the 
references therein. Mavinga [17] studied a problem with a nonlinear boundary condition from a variational point of view. Observe that in (1.1) we are dealing with two nonlinear terms that may also depend on the parameter $\lambda$. Our interest is in analysing the interplay between both nonlinearities. One of the main differences from problems with only one nonlinearity, either in the interior or at the boundary, is the regularity results (see theorem 2.4).

We study some possible bifurcations of branches of solutions as the parameter $\lambda$ varies. More specifically, we are interested in characterizing a bifurcation from infinity phenomenon, which localizes points in the parameter space where the branch of solutions becomes unbounded. We obtain sufficient conditions for having subcritical (on the left) or supercritical (on the right) bifurcations. Consequently, we also obtain the bifurcation nature of some classical results, such as the LandesmanLazer-type condition for the existence of solutions in the resonant case [15], and the anti-maximum principle $[4,10]$. We see that the bifurcation point of view also allows us to obtain a local maximum principle for some classes of strongly resonant problems.

Throughout this paper we shall assume that the nonlinearities $f$ and $g$ are sublinear at infinity with respect to the variable $u$, i.e.

$$
|f(\lambda, x, s)|=o(|s|), \quad|g(\lambda, x, s)|=o(|s|) \quad \text { as }|s| \rightarrow \infty .
$$

Hence, the nonlinearities in the interior and on the boundary are both asymptotically linear at infinity, since the dominant term for large values of $|u|$ is the linear term. Observe that problem (1.1) can be cast as a perturbation of the eigenvalue problem

$$
\left.\begin{array}{rlrl}
-\Delta u+u & =\mu m(x) u & & \text { in } \Omega, \\
\frac{\partial u}{\partial \nu} & =\mu \rho(x) u & & \text { on } \partial \Omega .
\end{array}\right\}
$$

We say that $\mu$ is a generalized eigenvalue if the linear problem (1.2) has a nontrivial solution. Note that the eigenvalue problem (1.2) includes as special cases the weighted Steklov eigenproblem (when $m \equiv 0$ and $\rho \not \equiv 0$ ) and the weighted Neumann eigenproblem (when $\rho \equiv 0$ and $m \not \equiv 0$ ).

In [17, theorem 2.1] the existence of a sequence of real eigenvalues $\mu_{n}:=\mu_{n}(m, \rho)$ of (1.2) such that $\mu_{n} \rightarrow \infty$ as $n \rightarrow \infty$ is proved, and each eigenvalue has a finitedimensional eigenspace. The eigenfunctions $\phi_{n}$ corresponding to these eigenvalues form a complete orthonormal family in the (proper) subspace of $H^{1}(\Omega)$. Moreover, the first eigenvalue, $\mu_{1}$, is simple, its associated eigenfunction, $\phi_{1}$, is strictly positive (or strictly negative) in $\Omega$ and the following inequality holds:

$$
\mu_{1}\left(\int_{\Omega} m(x) u^{2}+\int_{\partial \Omega} \rho(x) u^{2}\right) \leqslant \int_{\Omega}|\nabla u|^{2}+\int_{\Omega} u^{2} \quad \text { for all } u \in H^{1}(\Omega),
$$

where $\mu_{1}>0$ is the least eigenvalue for (1.2).

Observe that when $f=g=0$ the problem (1.1) becomes the linear problem (1.2). If the parameter $\lambda$ is not a generalized eigenvalue, then the only solution is the trivial one. On the other hand, if the parameter $\lambda$ is a generalized eigenvalue $\mu_{n}:=\mu_{n}(m, \rho)$ of (1.2), then (1.1) (with $f=g=0$ ) has infinitely many solutions. Indeed, any multiple of the eigenfunction $\phi_{n}$ is a solution. This phenomenon 
can be seen as the existence of an unbounded branch of solutions for $\lambda=\mu_{n}$. Bifurcation theory from infinity analyses how this unbounded branch of solutions is perturbed in the sublinear case, when the parameter $\lambda$ approaches a generalized eigenvalue.

The aim of this paper is to analyse some possible bifurcations of (weak) solutions as the parameter $\lambda$ varies. By a (weak) solution, we mean a solution $u \in H^{1}(\Omega)$ such that

$$
\int_{\Omega} \nabla u \nabla v+\int_{\Omega} u v=\lambda\left[\int_{\Omega} m(x) u v+\int_{\partial \Omega} \rho(x) u v\right]+\int_{\Omega} f(\lambda, x, u) v+\int_{\partial \Omega} g(\lambda, x, u) v
$$

for all $v \in H^{1}(\Omega)$.

The paper is organized as follows. In $\S 2$, we formulate the nonlinear problem (1.1) as a fixed-point problem in certain spaces, and use the tools of nonlinear functional analysis to prove the existence of solutions for all values of $\lambda \in \mathbb{R}$ except for the generalized eigenvalues. In $\S 3$, we show the existence of unbounded branches of solutions bifurcating from the generalized eigenvalues of odd multiplicity. The proof is based on the global bifurcation results of Rabinowitz [20,21] (see also [5-7]). Section 4 is devoted to the sub- and supercritical bifurcations from infinity. Moreover, in $\S 5$, we prove the existence of solutions for the problem at resonance under Landesman-Lazer-type conditions. Finally, $\S 6$, we analyse an anti-maximum principle.

\section{Non-resonance and fixed-point problems}

In this section, we prove the existence of a weak solution of (1.1) for all $\lambda \in \mathbb{R} \backslash$ $\left\{\mu_{i}\right\}_{i}$. We transform (1.1) into an equivalent fixed-point problem in $C^{0}(\bar{\Omega})$, and use compactness results, the Schaefer fixed-point theorem and regularity results to show that a weak solution of (1.1) also belongs to $C^{\alpha}(\bar{\Omega})$. One advantage of this technique is that it allows us to obtain more information on the resolvent operator of the linear problem associated with problem (1.1); that is, for any compact set of $\lambda$ far from the generalized eigenvalues, the norm of the resolvent operator is uniformly bounded in some appropriate spaces.

We assume that $(m, \rho) \in L^{\infty}(\Omega) \times L^{\infty}(\partial \Omega)$ and that $m$ and $\rho$ satisfy the following condition:

$$
\begin{aligned}
& m(x) \geqslant 0 \text { almost everywhere (a.e.) on } \Omega \text { and } \rho(x) \geqslant 0 \text { a.e. on } \partial \Omega \\
& \text { such that } \int_{\Omega} m(x) \mathrm{d} x+\int_{\partial \Omega} \rho(x) \mathrm{d} x \neq 0 .
\end{aligned}
$$

(We stress the fact that the weight-functions $m$ and $\rho$ may vanish on subsets of positive measure.)

The (nonlinear) perturbations $f: \mathbb{R} \times \Omega \times \mathbb{R} \rightarrow \mathbb{R}$ and $g: \mathbb{R} \times \partial \Omega \times \mathbb{R} \rightarrow \mathbb{R}$ satisfy the following conditions.

(C1) $f$ and $g$ are Carathéodory functions (i.e. measurable in $x$, and continuous with respect to $(\lambda, u) \in \mathbb{R} \times \mathbb{R})$. 
(C2) There exist $h_{1} \in L^{r}(\Omega)$ and $h_{2} \in L^{r^{\prime}}(\partial \Omega)$ (with $r>\frac{1}{2} N$ and $r^{\prime}>N-1$ ) and continuous functions $\Lambda_{1}, \Lambda_{2}: \mathbb{R} \rightarrow \mathbb{R}^{+}$and $\mathcal{U}_{1}, \mathcal{U}_{2}: \mathbb{R} \rightarrow \mathbb{R}^{+}$such that

$$
\begin{array}{ll}
|f(\lambda, x, s)| \leqslant \Lambda_{1}(\lambda) h_{1}(x) \mathcal{U}_{1}(s) & \text { for all }(\lambda, x, s) \in \mathbb{R} \times \Omega \times \mathbb{R} . \\
|g(\lambda, x, s)| \leqslant \Lambda_{2}(\lambda) h_{2}(x) \mathcal{U}_{2}(s) & \text { for all }(\lambda, x, s) \in \mathbb{R} \times \partial \Omega \times \mathbb{R} .
\end{array}
$$

We shall also assume the following conditions on the functions $\mathcal{U}_{1}$ and $\mathcal{U}_{2}$ :

(C3) $\lim _{|s| \rightarrow \infty} \mathcal{U}_{1}(s) / s=0$ and $\lim _{|s| \rightarrow \infty} \mathcal{U}_{2}(s) / s=0$.

Note that condition (C3) is satisfied in particular if $f, g: \mathbb{R} \times \bar{\Omega} \times \mathbb{R} \rightarrow \mathbb{R}$ are continuous, and

$$
\lim _{|s| \rightarrow \infty} \frac{f(\lambda, x, s)}{s}=0, \quad \lim _{|s| \rightarrow \infty} \frac{g(\lambda, x, s)}{s}=0
$$

uniformly for a.e. $x \in \partial \Omega$ and $\lambda$ on bounded intervals.

The following theorem states the existence of solutions for the non-resonance problem.

Theorem 2.1. Suppose that $f$ and $g$ satisfy (C1)-(C3). Then, for all $\lambda \in \mathbb{R} \backslash\left\{\mu_{i}\right\}_{i}$, there exists at least one solution of problem (1.1). Moreover, for each compact set $K \subset \mathbb{R} \backslash\left\{\mu_{i}\right\}_{i}$, there exists a constant $C=C(K)$ such that any solution of (1.1) is bounded in $C^{0}(\bar{\Omega})$ by $C$.

Before proving theorem 2.1, we first state and prove some relevant auxiliary results.

Consider first the linear problem

$$
\left.\begin{array}{rl}
-\Delta u+u=a(x) & \text { in } \Omega, \\
\frac{\partial u}{\partial \nu}=b(x) & \text { on } \partial \Omega .
\end{array}\right\}
$$

It is well known (see [12, p. 75]; [14, p. 162]; [18, p. 255]) that if $a \in L^{q}(\Omega)$ with $q>1$, then there exists a unique solution $u_{1} \in W^{2, q}(\Omega)$ for the following problem:

$$
\left.\begin{array}{rl}
-\Delta u_{1}+u_{1}=a & \text { in } \Omega, \\
\frac{\partial u_{1}}{\partial \nu}=0 & \text { on } \partial \Omega .
\end{array}\right\}
$$

We define the operator

$$
A: \operatorname{Dom}(A) \rightarrow L^{q}(\Omega)
$$

by

$$
A:=-\Delta+I,
$$

where

$$
\operatorname{Dom}(A):=\left\{u \in W^{2, q}(\Omega): \frac{\partial u}{\partial \nu}=0\right\} .
$$

It follows from the estimates in $[14$, p. 162] and $[18$, p. 255] that

$$
\left\|u_{1}\right\|_{W^{2, q}(\Omega)} \leqslant C\|a\|_{L^{q}(\Omega)} \quad \text { and } \quad u_{1}=A^{-1}(a) \in W^{2, q}(\Omega) .
$$


Moreover, the operator $A$ has an associated scale of interpolation-extrapolation spaces and, in particular, for each $p>1$, we have that $A: W^{1, p}(\Omega) \rightarrow W^{-1, p}(\Omega)$ is an isomorphism.

On the other hand, we use the boundary trace imbedding theorem,

$$
i: W^{1, p}(\Omega) \hookrightarrow L^{q}(\partial \Omega) \text { for any } p>1 \text { with } 1 \leqslant q \leqslant \frac{N p-p}{N-p},
$$

which is continuous, and compact for $1 \leqslant q<(N p-p) /(N-p)$ (see [1, p. 164]; [13, p. 344]), and the dual of the linear map $i$,

$$
i^{*}:\left(L^{q}(\partial \Omega)\right)^{*} \rightarrow\left(W^{1, p}(\Omega)\right)^{*},
$$

to get the embedding $L^{q^{*}}(\partial \Omega) \hookrightarrow W^{-1, p^{*}}(\Omega)$, where $\left(L^{q}(\partial \Omega)\right)^{*}=L^{q^{*}}(\partial \Omega)$ with $1 / q^{*}+1 / q=1$, and $\left(W^{1, p}(\Omega)\right)^{*}=W^{-1, p^{*}}(\Omega)$ with $1 / p^{*}+1 / p=1$. Therefore, choosing any $q^{\prime} \geqslant 1$, we get the embedding $L^{q^{\prime}}(\partial \Omega) \hookrightarrow W^{-1, p}(\Omega)$, which is continuous for $p^{*} \leqslant N q^{*} /(N-1)$ and compact for $p^{*}<N q^{*} /(N-1)$.

It follows that, for $b \in L^{q^{\prime}}(\partial \Omega)$ with $q^{\prime} \geqslant 1$, the unique solution, $u_{2}$, of

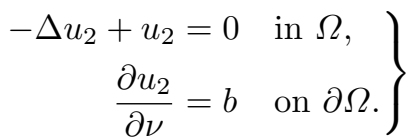

is in $W^{1, p}(\Omega)$ and $\left\|u_{2}\right\|_{W^{1, p}(\Omega)} \leqslant C\|b\|_{L^{q^{\prime}}(\partial \Omega)}$.

Using embedding theorems, we get that, for every $(a, b) \in L^{q}(\Omega) \times L^{q^{\prime}}(\partial \Omega)$ with $q>1$ and $q^{\prime} \geqslant 1$, the linear problem (2.2) has a unique solution $u \in W^{1, p}(\Omega)$ given by $u=u_{1}+u_{2}$, where

$$
p= \begin{cases}\min \left\{\frac{q N}{N-q}, \frac{q^{\prime} N}{N-1}\right\} & \text { for } 1<q<N, \\ \frac{q^{\prime} N}{N-1} & \text { for } q \geqslant N .\end{cases}
$$

Now, define the resolvent operator $S_{0}$ by

$$
S_{0}(a, b):=(u, \Gamma u)
$$

where $\Gamma u$ represents the trace of $u$. Note that, whenever $q<\frac{1}{2} N$ or $q^{\prime}<N-1$,

$$
p=\min \left\{\frac{q N}{N-q}, \frac{q^{\prime} N}{N-1}\right\}<N .
$$

On the other hand, if $q>\frac{1}{2} N$ and $q^{\prime}>N-1$, then $p>N$, and by Sobolev embeddings theorems we have that $W^{1, p}(\Omega)$ is embedded in $C^{\alpha}(\bar{\Omega})$ with $\alpha<$ $1-N / p$. Moreover, for each $u \in C^{\alpha}(\bar{\Omega})$ with $0 \leqslant \alpha<1$, the trace $\Gamma u \in C^{\alpha}(\partial \Omega)$ (and therefore, if needed, each $\left.u \in C^{\alpha}(\bar{\Omega})\right)$ can be rewritten as $(u, \Gamma u) \in C^{\alpha}(\bar{\Omega}) \times$ $C^{\alpha}(\partial \Omega)$.

In what follows, we shall show that any weak solution $u \in H^{1}(\Omega)$ of the nonlinear equation (1.1) lies in fact in $C^{\alpha}(\bar{\Omega})$. To accomplish this, we shall need several regularity results of the associated linear problem (2.2). The following lemma states the regularity of the solution of the linear problem. 
Lemma 2.2. If $N \geqslant 2$ and $a \in L^{q}(\Omega), b \in L^{q^{\prime}}(\partial \Omega)$ with $q>1$ and $q^{\prime} \geqslant 1$, then the linear problem (2.2) has a unique solution $u \in W^{1, p}(\Omega)$ satisfying

$$
\|u\|_{W^{1, p}(\Omega)} \leqslant C\left(\|a\|_{L^{q}(\Omega)}+\|b\|_{L^{q^{\prime}}(\partial \Omega)}\right),
$$

where (2.5) holds.

In particular, we have the following.

(i) If $q<\frac{1}{2} N$ or $q^{\prime}<N-1$ (which implies $1 \leqslant p<N$ ), then $u \in L^{p^{\prime}}(\Omega)$, $\Gamma u \in L^{p^{\prime \prime}}(\partial \Omega)$ for all $\left(p^{\prime}, p^{\prime \prime}\right) \in[1, N p /(N-p)] \times[1,(N-1) p /(N-p)]$. Moreover, the map $S_{0}: L^{q}(\Omega) \times L^{q^{\prime}}(\partial \Omega) \rightarrow L^{p^{\prime}}(\Omega) \times L^{p^{\prime \prime}}(\partial \Omega)$ is continuous for all $\left(p^{\prime}, p^{\prime \prime}\right) \in[1, N p /(N-p)] \times[1,(N-1) p /(N-p)]$, and compact for all $\left(p^{\prime}, p^{\prime \prime}\right) \in[1, N p /(N-p)) \times[1,(N-1) p /(N-p))$.

(ii) If $q=\frac{1}{2} N$ and $q^{\prime} \geqslant N-1$ (or $q \geqslant \frac{1}{2} N$ and $q^{\prime}=N-1$ ), which in both cases imply $p=N$, then $u \in L^{p^{\prime}}(\Omega)$ and $\Gamma u \in L^{p^{\prime}}(\partial \Omega)$ for any $p^{\prime} \geqslant 1$. Moreover, the map $S_{0}: L^{q}(\Omega) \times L^{q^{\prime}}(\partial \Omega) \rightarrow L^{p^{\prime}}(\Omega) \times L^{p^{\prime}}(\partial \Omega)$ is continuous and compact for $1 \leqslant p^{\prime}<\infty$.

(iii) If $q>\frac{1}{2} N$ and $q^{\prime}>N-1$ (which implies $p>N$ ), then $u \in C^{\alpha}(\bar{\Omega})$ with

$$
\|u\|_{C^{\alpha}(\bar{\Omega})} \leqslant C\left(\|a\|_{L^{q}(\Omega)}+\|b\|_{L^{q^{\prime}}(\partial \Omega)}\right)
$$

where $0<\alpha \leqslant 1-(N / p)$. Moreover, the map $S_{0}: L^{q}(\Omega) \times L^{q^{\prime}}(\partial \Omega) \rightarrow C^{\alpha}(\bar{\Omega}) \times$ $C^{\alpha}(\partial \Omega)$, with $0<\alpha<1-(N / p)$, is continuous and compact.

Proof. The existence, uniqueness and the estimate (2.6) are proved in a similar way as in $[2,5,14,19]$. To prove (i), we use the fact that if $1 \leqslant p<N$, then $W^{1, p}(\Omega)$ is compactly embedded in $L^{p^{\prime}}(\Omega)$ with $1 \leqslant p^{\prime}<N p /(N-p)$. Moreover, if $u \in W^{1, p}(\Omega)$, then $\Gamma u \in W^{1-1 / p, p}(\partial \Omega)$ (see [8, p. 315]), which is also compactly embedded in $L^{p^{\prime \prime}}(\partial \Omega)$ with $1 \leqslant p^{\prime \prime}<(N-1) p /(N-p)$. Similarly, one can use the Sobolev compact embedding theorem to prove (ii) and (iii).

The next corollary is a technical result that will be used for the regularity of a weak solution. Keeping the notation of the above lemma, we analyse where the minimum defining $p$ is attained (see (2.5)).

Corollary 2.3. Assume that $N \geqslant 2$, and let $(a, b)$ satisfy the conditions

$$
|a(x)| \leqslant h_{1}(x) w_{1}(x), \quad|b(x)| \leqslant h_{2}(x) w_{2}(x),
$$

where $h_{1} \in L^{r}(\Omega), w_{1} \in L^{s}(\Omega), h_{2} \in L^{r^{\prime}}(\partial \Omega), w_{2} \in L^{s^{\prime}}(\partial \Omega)$ with $r>\frac{1}{2} N, s>1$, $r^{\prime}>N-1$ and $s^{\prime} \geqslant 1$.

Let

$$
\frac{1}{q}=\frac{1}{r}+\frac{1}{s} \quad \text { and } \quad \frac{1}{q^{\prime}}=\frac{1}{r^{\prime}}+\frac{1}{s^{\prime}}
$$

where (2.5) holds.

Assume that $p<N$, then the following hold.

(i) If $p=q N /(N-q)$, then $q<\frac{1}{2} N$ and $S_{0}(a, b) \in L^{s+\delta}(\Omega) \times L^{p^{\prime \prime}}(\partial \Omega)$ with

$$
\delta=s\left(\frac{2}{N}-\frac{1}{r}\right)\left(\frac{1}{q}-\frac{2}{N}\right)^{-1}>0 \quad \text { and } \quad p^{\prime \prime}=\frac{(N-1) p}{N-p} .
$$


(ii) If $p=q^{\prime} N /(N-1)$, then $q^{\prime}<N-1$ and $S_{0}(a, b) \in L^{p^{\prime}}(\Omega) \times L^{s^{\prime}+\delta^{\prime}}(\partial \Omega)$ with

$$
\delta^{\prime}=s^{\prime}\left(\frac{1}{N-1}-\frac{1}{r^{\prime}}\right)\left(\frac{1}{q^{\prime}}-\frac{1}{N-1}\right)^{-1}>0 \quad \text { and } \quad p^{\prime}=\frac{N p}{N-p} .
$$

(iii) Furthermore, in any case $\left\|S_{0}(a, b)\right\| \leqslant C\left(\left\|w_{1}\right\|_{L^{s}(\Omega)}+\left\|w_{2}\right\|_{L^{s^{\prime}}(\partial \Omega)}\right)$.

Proof.

(i) Suppose that $p=q N /(N-q)$. Since, by assumption, $p<N$, one can see clearly that $q<\frac{1}{2} N$. From lemma 2.2,u $\in L^{p^{\prime}}(\Omega)$ for $p^{\prime}=N p /(N-p)$. Now, we need to prove that there exists $\delta>0$ such that $p^{\prime} \geqslant s+\delta$. Indeed, by the definitions of $p^{\prime}$, $p, q$ and taking into account that $r>\frac{1}{2} N$, we have that

$$
\frac{1}{p^{\prime}}=\frac{1}{p}-\frac{1}{N}=\frac{1}{q}-\frac{2}{N}=\frac{1}{r}+\frac{1}{s}-\frac{2}{N}<\frac{1}{s} .
$$

Choosing $\delta=p^{\prime}-s>0$, a direct calculation shows that

$$
\delta=s\left(\frac{2}{N}-\frac{1}{r}\right)\left(\frac{1}{q}-\frac{2}{N}\right)^{-1} .
$$

(ii) Suppose that $p=q^{\prime} N /(N-1)$. Since $p<N$, one can see clearly that $q^{\prime}<N-1$. From lemma 2.2, $\Gamma u \in L^{p^{\prime \prime}}(\partial \Omega)$ for $p^{\prime \prime}=(N-1) p /(N-p)$. We need to prove that there exists $\delta^{\prime}>0$ such that $p^{\prime \prime} \geqslant s^{\prime}+\delta^{\prime}$. By the definitions of $p^{\prime \prime}, p, q^{\prime}$ and taking into account that $r^{\prime}>N-1$, we have that

$$
\frac{1}{p^{\prime \prime}}=\frac{N}{(N-1) p}-\frac{1}{N-1}=\frac{1}{q^{\prime}}-\frac{1}{N-1}=\frac{1}{r^{\prime}}+\frac{1}{s^{\prime}}-\frac{1}{N-1}<\frac{1}{s^{\prime}} .
$$

Choosing $\delta^{\prime}=p^{\prime \prime}-s^{\prime}>0$, a direct calculation shows that

$$
\delta^{\prime}=s^{\prime}\left(\frac{1}{N-1}-\frac{1}{r^{\prime}}\right)\left(\frac{1}{q^{\prime}}-\frac{1}{N-1}\right)^{-1} .
$$

(iii) This is a consequence of lemma 2.2 (see (2.6)) and the Hölder inequality.

Now, we analyse the regularity result and prove that any weak solution $u \in$ $H^{1}(\Omega)$ is in fact in $C^{\alpha}(\bar{\Omega})$ for some for $0<\alpha<1$. The following result is one of the main differences from the problem with only one nonlinearity, either at the interior or at the boundary. It is a non-standard bootstrap argument. As in corollary 2.3 (see (2.6) and (2.5)), the regularity of a solution depends on the minimum of both the interior and the boundary regularities.

TheOrem 2.4. Assume that conditions (C1)-(C3) hold. For any $M>0$, if $u \in$ $H^{1}(\Omega)$ is a solution of the nonlinear problem (1.1) for some $|\lambda| \leqslant M$, then

$$
\|u\|_{C^{\alpha}(\bar{\Omega})} \leqslant C\left(1+\|u\|_{L^{s}(\Omega)}+\|\Gamma u\|_{L^{s^{\prime}}(\partial \Omega)}\right)
$$

for some positive $\alpha<1$, where $C=C(M), s=2 N /(N-2), s^{\prime}=2(N-1) /(N-2)$.

Proof. The proof is based on a four-step regularizing procedure.

In step 1 we start the procedure, letting $p, q, q^{\prime}$ initially be as in lemma 2.2; these will be made more precise later, in terms of the regularity of the nonlinear 
terms. Next we distinguish different cases of lemma 2.2. In step 2 we consider the $q>\frac{1}{2} N$ and $q^{\prime}>N-1$ case by using lemma 2.2(iii). In step 3, when $q=\frac{1}{2} N$ and $q^{\prime} \geqslant N-1$ (or $q \geqslant \frac{1}{2} N$ and $q^{\prime}=N-1$ ), we use lemma 2.2(ii). In step 4 we consider the case when $q<\frac{1}{2} N$ and $q^{\prime}<N-1$, invoking lemma 2.2(i). In turn, this step is divided into several parts. In part (a) we initialize the bootstrap argument. Part (b) is divided into two further sub-parts. We first distinguish where the minimum defining $p$ is attained (see (2.5)). We then consider the case where the minimum defining $p$ lies on the regularity of the 'interior' term, in other words $p=q N /(N-q) \leqslant q^{\prime} N /(N-1)$. We then consider the minimum defining $p$, which lies on the regularity of the 'boundary' term, which means $p=q^{\prime} N /(N-1) \leqslant$ $q N /(N-q)$. We prove that, in any case, this minimum can be 'raised' at most in the combination of those two sub-parts. In part (c) we iterate this procedure for $q=q_{i}<\frac{1}{2} N$ or $q^{\prime}=q_{j}^{\prime}<N-1, i, j \geqslant 0$. This part in turn is also divided into two sub-parts: we consider first the case where $q_{i}<\frac{1}{2} N$ for $i \geqslant 0$, and then the case where $q_{j}^{\prime}<N-1$ for $j \geqslant 0$. We conclude that, in a finite number of iterations, which may be different for the nonlinearity at the interior and the nonlinearity at the boundary, the corresponding $q_{i_{0}} \geqslant \frac{1}{2} N$, and also $q_{j_{0}}^{\prime} \geqslant N-1$. This situation corresponds to the case treated in step 3, which ends the proof.

Now, let us carry out the procedure.

STEP 1. Using conditions (C1)-(C3) and the fact that $|\lambda| \leqslant M$, we have that $|\lambda m(x) u+f(\lambda, x, u)| \leqslant C_{1}\left(1+h_{1}(x)\right)(1+|u(x)|)$ and $|\lambda \rho(x) u+g(\lambda, x, u)| \leqslant C_{2}(1+$ $\left.h_{2}(x)\right)(1+|u(x)|)$, where $C_{1}$ and $C_{2}$ are constants independent of $u$. Therefore,

$$
\begin{aligned}
-\Delta u+u & =a(x) \quad \text { in } \Omega, \\
\frac{\partial u}{\partial \nu} & =b(x) \quad \text { on } \partial \Omega,
\end{aligned}
$$

with $|a(x)| \leqslant C_{1}\left(1+h_{1}(x)\right)(1+|u(x)|)$ and $|b(x)| \leqslant C_{2}\left(1+h_{2}(x)\right)(1+|u(x)|)$. By conditions (C2), $1+h_{1}(x) \in L^{r}(\Omega)$ and $1+h_{2}(x) \in L^{r^{\prime}}(\partial \Omega)$ with $r>\frac{1}{2} N$ and $r^{\prime}>N-1$. Since $u \in H^{1}(\Omega)$, we have $u \in L^{s}(\Omega)$ and $\Gamma u \in L^{s^{\prime}}(\partial \Omega)$ with $s=2 N /(N-2)$ and $s^{\prime}=2(N-1) /(N-2)$. From Hölder's inequality, $(a, b) \in$ $L^{q}(\Omega) \times L^{q^{\prime}}(\partial \Omega)$ with

$$
\frac{1}{q}=\frac{1}{r}+\frac{1}{s} \quad \text { and } \quad \frac{1}{q^{\prime}}=\frac{1}{r^{\prime}}+\frac{1}{s^{\prime}} .
$$

Step 2. From lemma 2.2(iii), we have that if $q>\frac{1}{2} N$ and $q^{\prime}>N-1$, then $u \in C^{\alpha}(\bar{\Omega})$ with

$$
\begin{aligned}
\|u\|_{C^{\alpha}(\bar{\Omega})} & \leqslant C\left(\|a\|_{L^{q}(\Omega)}+\|b\|_{L^{q^{\prime}}(\partial \Omega)}\right) \\
& \leqslant C\left(1+\|u\|_{L^{s}(\Omega)}+\|\Gamma u\|_{L^{s^{\prime}}(\partial \Omega)}\right),
\end{aligned}
$$

which completes this step of the proof.

STEP 3. If $q=\frac{1}{2} N$ and $q^{\prime} \geqslant N-1$ (or $q \geqslant \frac{1}{2} N$ and $q^{\prime}=N-1$ ), from lemma 2.2(ii) we obtain that $(u, \Gamma u)=S_{0}(a, b) \in L^{p^{\prime}}(\Omega) \times L^{p^{\prime}}(\partial \Omega)$ for any $p^{\prime} \in[1, \infty)$. Consequently, $(a, b) \in L^{r-\varepsilon}(\Omega) \times L^{r^{\prime}-\varepsilon}(\partial \Omega)$ with $r-\varepsilon>\frac{1}{2} N$ and $r^{\prime}-\varepsilon>N-1$, and, finally, from lemma 2.2 (iii) we deduce that $u \in C^{\alpha}(\bar{\Omega})$, which ends this step of the proof. 
Step 4. Assume that $q<\frac{1}{2} N$ or $q^{\prime}<N-1$. Then, from lemma 2.2(i), we deduce that $u \in W^{1, p}(\Omega)$ and

$$
(u, \Gamma u)=S_{0}(a, b) \in L^{p^{\prime}}(\Omega) \times L^{p^{\prime \prime}}(\partial \Omega),
$$

where $p^{\prime}=N p /(N-p)$ and $p^{\prime \prime}=(N-1) p /(N-p)$ with $p=\min \{q N /(N-$ q), $\left.q^{\prime} N /(N-1)\right\}<N$.

(a) Let us define $s_{0}=s, q_{0}=q, s_{0}^{\prime}=s^{\prime}, q_{0}^{\prime}=q^{\prime}, p_{00}=p, p_{00}^{\prime}=p^{\prime}$ and $p_{00}^{\prime \prime}=p^{\prime \prime}$. With this notation,

$$
p_{00}=\min \left\{\frac{q_{0} N}{N-q_{0}}, \frac{q_{0}^{\prime} N}{N-1}\right\}<N .
$$

Let us define, in a general setting, $u \in W^{1, p_{i j}}(\Omega)$ and

$$
(u, \Gamma u)=S_{0}(a, b) \in L^{p_{i j}^{\prime}}(\Omega) \times L^{p_{i j}^{\prime \prime}}(\partial \Omega),
$$

where

$$
p_{i j}=\min \left\{\frac{q_{i} N}{N-q_{i}}, \frac{q_{j}^{\prime} N}{N-1}\right\} \quad \text { for } 1<q_{i}<N, \quad p_{i j}=\frac{q_{j}^{\prime} N}{N-1} \quad \text { for } q_{i} \geqslant N,
$$

and if $p_{i j}<N$,

$$
p_{i, j}^{\prime}=\frac{N p_{i, j}}{N-p_{i, j}} \quad \text { and } \quad p_{i, j}^{\prime \prime}=\frac{(N-1) p_{i, j}}{N-p_{i, j}} \quad \text { for } i, j=0,1, \ldots
$$

(b) Assume $q_{i}<\frac{1}{2} N$ or $q_{j}^{\prime}<N-1$ (which implies $1 \leqslant p_{i j}<N$ ) throughout part (b). We shall prove, in at most two iterations, that $u \in W^{1, p_{i+1, j+1}}(\Omega)$ with $p_{i+1, j+1}>p_{i, j}$.

(1) Assume that $p_{i j}=q_{i} N /\left(N-q_{i}\right)$. Then, from corollary 2.3(i) it follows that $u \in L^{s_{i+1}}(\Omega)$ with $s_{i+1}=s_{i}+\delta_{i}$, and

$$
\delta_{i}=s_{i}\left(\frac{2}{N}-\frac{1}{r}\right)\left(\frac{1}{q_{i}}-\frac{2}{N}\right)^{-1}>0 .
$$

Therefore, $(a, b) \in L^{q_{i+1}}(\Omega) \times L^{q_{j}^{\prime}}(\partial \Omega)$ with

$$
\frac{1}{q_{i+1}}=\frac{1}{r}+\frac{1}{s_{i+1}}
$$

and $q_{i+1}>q_{i}$. Moreover, from lemma 2.2, $u \in W^{1, p_{i+1, j}}(\Omega)$.

If $q_{i+1} \geqslant \frac{1}{2} N$ and $q_{j}^{\prime}<N-1$, then $p_{i+1, j}=q_{j}^{\prime} N /(N-1)<N$ and we shall skip to part (2).

On the other hand, if $q_{i+1}<\frac{1}{2} N$, then $p_{i+1, j}<N$ and, moreover, $(u, \Gamma u)=$ $S_{0}(a, b) \in L^{p_{i+1, j}^{\prime}}(\Omega) \times L^{p_{i+1, j}^{\prime \prime}}(\partial \Omega)$.

To conclude this sub-step, we can assert that $u \in W^{1, p_{i+1, j}}(\Omega)$, with $p_{i+1, j} \geqslant$ $p_{i j}$. Moreover,

$$
\text { if } \frac{q_{i} N}{N-q_{i}}<\frac{q_{j}^{\prime} N}{N-1}, \quad \text { then } p_{i+1, j}>p_{i j}
$$


and

if $\frac{q_{i} N}{N-q_{i}}=\frac{q_{j}^{\prime} N}{N-1}$,

$$
\text { then } p_{i j}=p_{i+1, j}=\frac{q_{j}^{\prime} N}{N-1}<\frac{q_{i+1} N}{N-q_{i+1}} \text { and } p_{i+1, j}<N \text {. }
$$

The situation in (2.9) is treated in (2), where we shall prove that $p_{i+1, j+1}>$ $p_{i+1, j}$.

(2) Assume that $p_{i j}=q_{j}^{\prime} N / N-1$. Then, from corollary 2.3(ii) it follows that $\Gamma u \in L^{s_{j+1}^{\prime}}(\partial \Omega)$ with $s_{j+1}^{\prime}=s_{j}^{\prime}+\delta_{j}^{\prime}$ and

$$
\delta_{j}^{\prime}=s_{j}^{\prime}\left(\frac{1}{N-1}-\frac{1}{r^{\prime}}\right)\left(\frac{1}{q_{j}^{\prime}}-\frac{1}{N-1}\right)^{-1}>0 .
$$

Therefore, $(a, b) \in L^{q_{i}}(\Omega) \times L^{q_{j+1}^{\prime}}(\partial \Omega)$ with

$$
\frac{1}{q_{j+1}^{\prime}}=\frac{1}{r^{\prime}}+\frac{1}{s_{j+1}^{\prime}} \quad \text { and } \quad q_{j+1}^{\prime}>q_{j}^{\prime} .
$$

Moreover, from lemma 2.2, $u \in W^{1, p_{i, j+1}}(\Omega)$.

If $q_{j+1}^{\prime}<N-1$, then $p_{i, j+1}<N$, and, moreover, $S_{0}(a, b) \in L^{p_{i, j+1}^{\prime}}(\Omega) \times$ $L^{p_{i, j+1}^{\prime \prime}}(\partial \Omega)$. Consequently, $u \in W^{1, p_{i, j+1}}(\Omega)$, with $p_{i, j+1} \geqslant p_{i j}$. Moreover,

$$
\text { if } \frac{q_{j}^{\prime} N}{N-1}<\frac{q_{i} N}{N-q_{i}}, \quad \text { then } p_{i, j+1}>p_{i j},
$$

and

$$
\text { if } \begin{aligned}
\frac{q_{j}^{\prime} N}{N-1}=\frac{q_{i} N}{N-q_{i}}, \\
\quad \text { then } p_{i j}=p_{i, j+1}=\frac{q_{i} N}{N-q_{i}}<\frac{q_{j+1}^{\prime} N}{N-1} \text { and } p_{i, j+1}<N .
\end{aligned}
$$

If (2.11) holds, we return to sub-step (1) for $p_{i, j+1}$ and observe that $p_{i+1, j+1}>$ $p_{i, j+1}$ (see $\left.(2.8)\right)$.

Summarizing, in (1) and (2) of part (b) we check where the minimum defining $p=p_{i j}=\min \left\{q_{i} N /\left(N-q_{i}\right), q_{j}^{\prime} N /(N-1)\right\}$ is attained, and then we prove that, in any case, this minimum can be raised at most in the combination of both (1) and (2), proving that $u \in W^{1, p_{i+1, j+1}}(\Omega)$ with $p_{i+1, j+1}>p_{i, j}$.

(c) Iterating this procedure, we shall prove that, in a finite number of steps, we get

$$
(a, b) \in L^{q_{i_{0}}}(\Omega) \times L^{q_{j_{0}}^{\prime}}(\partial \Omega) \quad \text { with } q_{i_{0}} \geqslant \frac{1}{2} N \text { and } q_{j_{0}}^{\prime} \geqslant N-1 .
$$

(1) To ascertain (2.12) when $q_{i}<\frac{1}{2} N$, let us set

$$
\frac{1}{s_{i}}=\frac{1}{q_{i}}-\frac{1}{r}
$$


and define

$$
\delta_{i}=s_{i}\left(\frac{2}{N}-\frac{1}{r}\right)\left(\frac{1}{q_{i}}-\frac{2}{N}\right)^{-1}>0, \quad s_{i+1}=s_{i}+\delta_{i}, \quad \frac{1}{q_{i+1}}=\frac{1}{r}+\frac{1}{s_{i+1}} .
$$

Observe that $s_{i+1}>s_{i}$ and $q_{i+1}>q_{i}$.

Consider the sequence $\left\{q_{i}\right\}$ with $q_{i}<\frac{1}{2} N$. If $\left\{q_{i}\right\}$ is a infinite set (observe that $\left\{q_{i}\right\}_{i}$ is an increasing sequence and $1<q_{i}<\frac{1}{2} N$ for all $i \in \mathbb{N}$ ), then there exists $\lim _{i \rightarrow \infty} q_{i}=: q_{\infty}$, and $1<q_{\infty} \leqslant \frac{1}{2} N$.

Moreover, $\left\{s_{i}\right\}_{i}$ is an increasing sequence, and, by definition,

$$
\frac{1}{s_{i}}=\frac{1}{q_{i}}-\frac{1}{r}>0 \text {. }
$$

Therefore, there exists

$$
\lim _{i \rightarrow \infty} \frac{1}{s_{i}}=\frac{1}{q_{\infty}}-\frac{1}{r} \geqslant \frac{2}{N}-\frac{1}{r}>0 .
$$

Consequently, there exists $\lim _{i \rightarrow \infty} s_{i}=: s_{\infty}$, and

$$
1<\frac{r}{r-1} \leqslant s_{\infty} \leqslant\left(\frac{2}{N}-\frac{1}{r}\right)^{-1}<\infty .
$$

Also, by definition, $\delta_{i}=s_{i+1}-s_{i}$. Then, there exists $\lim _{i \rightarrow \infty} \delta_{i}=0$. Let us set $\delta_{\infty}=\lim _{i \rightarrow \infty} \delta_{i}=0$.

If $q_{\infty}<\frac{1}{2} N$, then, by definition,

$$
\delta_{\infty}=s_{\infty}\left(\frac{2}{N}-\frac{1}{r}\right)\left(\frac{1}{q_{\infty}}-\frac{2}{N}\right)^{-1}>0
$$

which contradicts $\delta_{\infty}=0$. Then, necessarily, $q_{\infty}=\frac{1}{2} N$.

Now, using the definition of $\delta_{i}$ we can write

$$
s_{i}=\delta_{i}\left(\frac{1}{q_{i}}-\frac{2}{N}\right)\left(\frac{2}{N}-\frac{1}{r}\right)^{-1} \rightarrow 0 \quad \text { as } i \rightarrow \infty,
$$

which again contradicts (2.13). Consequently, $\left\{q_{i}\right\}$ is a finite set (for example, $\left.\left\{q_{0}, q_{1}, \ldots, q_{i_{0}-1}\right\}\right)$, and $q_{i_{0}} \geqslant \frac{1}{2} N$, completing this part of the proof.

(2) On the other hand, assume $q_{j}^{\prime}<N-1$. Let us set

$$
\frac{1}{s_{j}^{\prime}}=\frac{1}{q_{j}^{\prime}}-\frac{1}{r^{\prime}}
$$

and define

$$
\begin{gathered}
\delta_{j}^{\prime}=s_{j}^{\prime}\left(\frac{1}{N-1}-\frac{1}{r^{\prime}}\right)\left(\frac{1}{q_{j}^{\prime}}-\frac{1}{N-1}\right)^{-1}>0, \quad s_{j+1}^{\prime}=s_{j}^{\prime}+\delta_{j}^{\prime}, \\
\frac{1}{q_{j+1}^{\prime}}=\frac{1}{r^{\prime}}+\frac{1}{s_{j+1}^{\prime}} .
\end{gathered}
$$


Observe that $s_{j+1}^{\prime}>s_{j}^{\prime}$ and $q_{j+1}^{\prime}>q_{j}^{\prime}$. Let us consider the sequence $\left\{q_{j}^{\prime}\right\}$, when $q_{j}^{\prime}<N-1$. If $\left\{q_{j}^{\prime}\right\}$ is an infinite sequence, we observe that $\left\{q_{j}^{\prime}\right\}_{j}$ is increasing, and $q_{j}^{\prime}<N-1$ for all $j \in \mathbb{N}$. Then there exists $\lim _{j \rightarrow \infty} q_{j}^{\prime}=: q_{\infty}^{\prime}$, and $1<q_{\infty}^{\prime} \leqslant N-1$.

Moreover, $\left\{s_{j}^{\prime}\right\}_{j}$ is an increasing sequence, and, by definition,

$$
\frac{1}{s_{j}^{\prime}}=\frac{1}{q_{j}^{\prime}}-\frac{1}{r^{\prime}}
$$

Therefore, there exists

$$
\lim _{j \rightarrow \infty} \frac{1}{s_{j}^{\prime}}=\frac{1}{q_{\infty}^{\prime}}-\frac{1}{r^{\prime}} \geqslant \frac{2}{N}-\frac{1}{r}>0 .
$$

Consequently, there exists $\lim _{j \rightarrow \infty} s_{j}^{\prime}=: s_{\infty}^{\prime}$, and

$$
1<\frac{r^{\prime}}{r^{\prime}-1} \leqslant s_{\infty}^{\prime} \leqslant \frac{1}{1 /(N-1)-1 / r^{\prime}}<\infty .
$$

Also by definition, $\delta_{j}^{\prime}=s_{j+1}^{\prime}-s_{j}^{\prime}$. Thus, there exists $\lim _{j \rightarrow \infty} \delta_{j}^{\prime}=0$. Let us set $\delta_{\infty}^{\prime}=\lim _{j \rightarrow \infty} \delta_{j}^{\prime}=0$.

We proceed as before. If $q_{\infty}^{\prime}<N-1$, then, by definition,

$$
\delta_{\infty}^{\prime}=s_{\infty}\left(\frac{1}{N-1}-\frac{1}{r^{\prime}}\right)\left(\frac{1}{q_{\infty}^{\prime}}-\frac{1}{N-1}\right)^{-1}>0
$$

which is a contradiction, so $q_{\infty}^{\prime}=N-1$. Now, from the definition of $\delta_{j}^{\prime}$ we can write

$$
s_{j}^{\prime}=\delta_{j}^{\prime}\left(\frac{1}{q_{j}^{\prime}}-\frac{1}{N-1}\right)\left(\frac{1}{N-1}-\frac{1}{r}\right)^{-1} \rightarrow 0 \quad \text { as } j \rightarrow \infty,
$$

which contradicts (2.14). Consequently, $\left\{q_{j}^{\prime}\right\}$ is a finite set, for example, $\left\{q_{0}^{\prime}, q_{1}^{\prime}, \ldots, q_{j_{0}-1}^{\prime}\right\}$, and $q_{j_{0}}^{\prime} \geqslant N-1$, ending this part of the proof.

Combining sub-steps (1) and (2) of (c), we achieve (2.12), which, combined with step 3, completes the proof.

In the next lemmas, we derive some spectral properties of the resolvent operator $S_{0}$ in $L^{2}(\Omega) \times L^{2}(\partial \Omega)$. Throughout this paper, we shall consider the inner product on $L^{2}(\Omega) \times L^{2}(\partial \Omega)$ defined by

$$
\langle(u, v),(w, z)\rangle:=\int_{\Omega} u w+\int_{\partial \Omega} v z .
$$

Lemma 2.5. The resolvent operator $S_{0}: L^{2}(\Omega) \times L^{2}(\partial \Omega) \rightarrow L^{2}(\Omega) \times L^{2}(\partial \Omega)$ is a linear, self-adjoint, positive and compact operator. If we denote its eigenvalues by $\left\{\tau_{i}\right\}$, then $\tau_{i}=1 / \mu_{i}(1,1)$, where $\mu_{i}=\mu_{i}(m, \rho)$ are the generalized eigenvalues.

Proof. Let $a_{i} \in L^{2}(\Omega)$ and $b_{i} \in L^{2}(\partial \Omega)$, and let $v_{i}$ be the corresponding solution of $-\Delta v_{i}+v_{i}=a_{i}, \partial v_{i} / \partial \nu=b_{i}$ with $i=1,2$. The linearity of $S_{0}$ follows from the definition of the resolvent operator and the linearity of the trace operator. 
By a weak formulation of the problem, we have that

$$
\begin{aligned}
\left\langle\left(a_{1}, b_{1}\right), S_{0}\left(a_{2}, b_{2}\right)\right\rangle & =\left\langle\left(a_{1}, b_{1}\right),\left(v_{2}, \Gamma v_{2}\right)\right\rangle \\
& =\int_{\Omega} a_{1} v_{2}+\int_{\partial \Omega} b_{1} \Gamma v_{2}=\int_{\Omega} \nabla v_{1} \nabla v_{2}+\int_{\Omega} v_{1} v_{2} \\
& =\int_{\Omega} a_{2} v_{1}+\int_{\partial \Omega} b_{2} \Gamma v_{1}=\left\langle S_{0}\left(a_{1}, b_{1}\right),\left(a_{2}, b_{2}\right)\right\rangle,
\end{aligned}
$$

which implies that $S_{0}$ is self-adjoint.

Moreover, by the maximum principle, $S_{0}$ is a positive operator in the sense that if $a, b \geqslant 0$, then $u \geqslant 0$, where $(u, \Gamma u)=S_{0}(a, b)$ (see [3]). The compactness of $S_{0}$ follows from lemma 2.2.

LEMma 2.6. For any $\lambda \in \mathbb{R}$ with $\lambda \notin\left\{\mu_{i}\right\}_{i}$, define the operator $S_{\lambda}: L^{2}(\Omega) \times$ $L^{2}(\partial \Omega) \rightarrow L^{2}(\Omega) \times L^{2}(\partial \Omega)$ by $S_{\lambda}(a, b):=(u, \Gamma u)$, where $u \in H^{1}(\Omega)$ is the unique solution of

$$
\left.\begin{array}{rlrl}
-\Delta u+u & =\lambda m(x) u+a(x) & & \text { in } \Omega, \\
\frac{\partial u}{\partial \nu} & =\lambda \rho(x) u+b(x) & & \text { on } \partial \Omega .
\end{array}\right\}
$$

Then $S_{\lambda}$ is self-adjoint, continuous and compact. Moreover, if $q>\frac{1}{2} N$ and $q^{\prime}>$ $N-1$, then $S_{\lambda}: L^{q}(\Omega) \times L^{q^{\prime}}(\partial \Omega) \rightarrow C^{0}(\bar{\Omega}) \times C^{0}(\partial \Omega)$ is continuous and compact and, for any compact set $K \subset \mathbb{R} \backslash\left\{\mu_{i}\right\}$, the norm of $S_{\lambda}: L^{q}(\Omega) \times L^{q^{\prime}}(\partial \Omega) \rightarrow$ $C^{0}(\bar{\Omega}) \times C^{0}(\partial \Omega)$ is uniformly bounded for $\lambda \in K$. Also, $\left\|S_{\lambda}\right\| \rightarrow \infty$ as $\lambda \rightarrow \mu_{i}$ for some $i$.

Proof. Observe that $S_{\lambda}(a, b)=(u, \Gamma u)=S_{0}(\lambda m(x) u+a, \lambda \rho(x) u+b)$.

Hence,

$$
\begin{aligned}
S_{\lambda}(a, b) & =(u, \Gamma u) \\
& =\lambda S_{0}(m(x) u, \rho u)+S_{0}(a, b) \\
& =\lambda S_{0} \circ \Theta(u, \Gamma u)+S_{0}(a, b),
\end{aligned}
$$

where the map $\Theta: L^{2}(\Omega) \times L^{2}(\partial \Omega) \rightarrow L^{2}(\Omega) \times L^{2}(\partial \Omega)$ is defined as $\Theta(u, \Gamma u)=$ $(m(x) u, \rho(x) u)$.

Hence,

$$
(u, \Gamma u)-\lambda S_{0} \circ \Theta(u, \Gamma u)=S_{0}(a, b)
$$

or, equivalently,

$$
\left(I-\lambda S_{0} \circ \Theta\right)(u, \Gamma u)=S_{0}(a, b)
$$

Then,

$$
\left(I-\lambda S_{0} \circ \Theta\right) S_{\lambda}(a, b)=S_{0}(a, b) .
$$

Since $\lambda \notin\left\{\mu_{i}\right\}_{i}$, it follows from the Fredholm alternative theorem that $I-\lambda S_{0} \circ \Theta$ is invertible. Thus,

$$
S_{\lambda}=\left(I-\lambda S_{0} \circ \Theta\right)^{-1} \circ S_{0}
$$

Using the properties of $S_{0}$ in lemma 2.5, we can now prove this lemma. 
Note that $u \in H^{1}(\Omega)$ is a solution of (1.1) if and only if $(u, \Gamma u)$ is a fixed point of

$$
\begin{aligned}
(u, \Gamma u) & =\lambda S_{0} \circ \Theta(u, \Gamma u)+S_{0}(f(\lambda, \cdot, u), g(\lambda, \cdot, \Gamma u)) \\
& =S_{\lambda}(f(\lambda, \cdot, u), g(\lambda, \cdot, \Gamma u)) .
\end{aligned}
$$

The following lemma concerns an a priori estimate that is needed later.

Lemma 2.7. Assume that (C1)-(C3) hold. Then the Nemytskii operator

$$
\begin{aligned}
\mathcal{N}: C^{0}(\bar{\Omega}) \times C^{0}(\partial \Omega) \rightarrow L^{r}(\Omega) & \times L^{r^{\prime}}(\partial \Omega) \\
& \text { given by } \mathcal{N}(u, \Gamma u)=(f(\lambda, \cdot, u), g(\lambda, \cdot, \Gamma u))
\end{aligned}
$$

with $r>\frac{1}{2} N$ and $r^{\prime}>N-1$ is well defined and continuous. Moreover, for each $M>0$ and $\varepsilon>0$, there exists a constant $C=C(\varepsilon, M)$ such that

$$
\|f(\lambda, \cdot, u)\|_{L^{r}(\Omega)}+\|g(\lambda, \cdot, \Gamma u)\|_{L^{r^{\prime}}(\partial \Omega)} \leqslant \varepsilon\|u\|_{C^{0}(\bar{\Omega})}+C
$$

for all $u \in C^{0}(\bar{\Omega}),|\lambda| \leqslant M$.

In particular, the map $C^{0}(\bar{\Omega}) \times C^{0}(\partial \Omega) \ni(u, \Gamma u) \rightarrow S_{\lambda}(f(\lambda, \cdot, u), g(\lambda, \cdot, \Gamma u)) \in$ $C^{0}(\bar{\Omega}) \times C^{0}(\partial \Omega)$ is continuous and compact for all $\lambda \in \mathbb{R} \backslash\left\{\mu_{i}\right\}$.

Proof. It follows from the bounds of $f$ and $g$ in condition (C1) that the map $\mathcal{N}$ is well defined. The continuity follows from the continuity of $f$ and $g$ with respect to the last variable, the bounds of $f, g$ given by $(\mathrm{C} 2)$ and the dominated convergence theorem. The inequality (2.19) follows from (C3), which implies that for each $\varepsilon>0$ we have the inequality $\left|U_{i}(s)\right| \leqslant \varepsilon s+C$ for $i=1,2$ and some constant $C=C(\varepsilon)$, and the fact that the function $\Lambda(\lambda)$ is continuous.

Now, we are ready to prove the existence of solutions for the non-resonance problem.

Proof of theorem 2.1. Consider the compact set $K \subset \mathbb{R} \backslash\left\{\mu_{i}\right\}$. By lemma 2.6, there exists a constant $\tilde{C}=\tilde{C}(K)$ such that the norm of $S_{\lambda}: L^{r}(\Omega) \times L^{r^{\prime}}(\partial \Omega) \rightarrow C^{0}(\bar{\Omega}) \times$ $C^{0}(\partial \Omega)$ is bounded by $\tilde{C}$ for all $\lambda \in K$. Now, we shall show that the conditions of the Schaefer fixed-point theorem are satisfied. Let $\delta \in[0,1]$ and $(u, \Gamma u) \in C^{0}(\bar{\Omega}) \times$ $C^{0}(\partial \Omega)$ be such that

$$
(u, \Gamma u)=\delta S_{\lambda}(f(\lambda, \cdot, u), g(\lambda, \cdot, \Gamma u))
$$

for some $\lambda \in K$. By lemma 2.7, we have that the map

$$
C^{0}(\bar{\Omega}) \times C^{0}(\partial \Omega) \ni(u, \Gamma u) \rightarrow S_{\lambda}(f(\lambda, \cdot, u), g(\lambda, \cdot, \Gamma u)) \in C^{0}(\bar{\Omega}) \times C^{0}(\partial \Omega)
$$

is continuous and compact for $\lambda \in K$. We now need to show that $(u, \Gamma u)$ is bounded in $C^{0}(\bar{\Omega}) \times C^{0}(\partial \Omega)$. It follows from (2.20) and lemma 2.6 that

$$
\|(u, \Gamma u)\|_{C^{0}(\bar{\Omega}) \times C^{0}(\partial \Omega)} \leqslant \tilde{C}\|(f(\lambda, \cdot, u), g(\lambda, \cdot, \Gamma u))\|_{L^{r}(\Omega) \times L^{r^{\prime}}(\partial \Omega)} .
$$

Using (2.19), we get that

$$
\|(u, \Gamma u)\|_{C^{0}(\bar{\Omega}) \times C^{0}(\partial \Omega)} \leqslant \tilde{C}\left[\varepsilon\|u\|_{C^{0}(\bar{\Omega})}+C(\varepsilon, K)\right] .
$$


Choosing $\varepsilon$ small enough such that $1-\tilde{C} \varepsilon \geqslant \frac{1}{2}$, we get that $\|(u, \Gamma u)\|_{C^{0}(\bar{\Omega}) \times C^{0}(\partial \Omega)} \leqslant$ $2 \tilde{C} C$. Hence, $(u, \Gamma u)$ is bounded in $C^{0}(\Omega) \times C^{0}(\partial \Omega)$ independently of $\delta$. Then, by the Schaefer fixed point theorem (see [11]), the operator $C^{0}(\bar{\Omega}) \times C^{0}(\partial \Omega) \ni$ $(u, \Gamma u) \rightarrow S_{\lambda}(f(\lambda, \cdot, u), g(\lambda, \cdot, \Gamma u)) \in C^{0}(\bar{\Omega}) \times C^{0}(\partial \Omega)$ has a fixed point $(u, \Gamma u) \in$ $C^{0}(\bar{\Omega}) \times C^{0}(\partial \Omega)$. Due to lemmas 2.7 and $2.2(\mathrm{iii})$, we have that $u \in C^{\alpha}(\bar{\Omega})$. Equivalently, (1.1) has at least one solution $u \in C^{\alpha}(\bar{\Omega})$. The proof is complete.

\section{Unbounded branches of solutions}

In this section, we use the global bifurcation results of Rabinowitz [20,21] to show the existence of unbounded branches of solutions bifurcating from generalized eigenvalues of odd multiplicity. From theorem 2.1 and lemma 2.6 we know that, when the value of $\lambda$ is bounded away from the generalized eigenvalues, solutions of (1.1) are bounded uniformly in $\lambda$ and, furthermore, the norm of the resolvent operator of the linear problem associated with (1.1) blows up to infinity when $\lambda$ approaches a generalized eigenvalue. So, we can expect the existence of branches of solutions that diverge to infinity in a certain norm when the parameter tends to a generalized eigenvalue. We shall first analyse some necessary conditions for having solutions blowing up in the $C^{0}(\bar{\Omega})$ norm.

Proposition 3.1. Let $\left\{\lambda_{n}\right\}$ be a convergent sequence of real numbers for which there exist the corresponding solutions $u_{n}$ of (1.1) with $\left\|u_{n}\right\|_{C^{0}(\bar{\Omega})} \rightarrow \infty$ as $n \rightarrow \infty$. Then there exists a generalized eigenvalue $\mu_{i}$ such that $\lambda_{n} \rightarrow \mu_{i}$ and, for any subsequence of $\left\{u_{n}\right\}$, there exists a subsequence $\left\{u_{n_{k}}\right\}$ such that

$$
\frac{u_{n_{k}}}{\left\|u_{n_{k}}\right\|_{C^{0}(\bar{\Omega})}} \rightarrow \phi_{i} \quad \text { in } C^{\beta}(\bar{\Omega})
$$

for some $\beta>0$, where $\phi_{i}$ is an eigenfunction associated with the eigenvalue $\mu_{i}$.

Proof. Let $v_{n}:=u_{n} /\left\|u_{n}\right\|_{C^{0}(\bar{\Omega})}$. By the a priori estimate (2.7) and the fact that $\left\|u_{n}\right\|_{C^{0}(\bar{\Omega})} \rightarrow \infty$ we have that $\left\|v_{n}\right\|_{C^{\alpha}(\bar{\Omega})}$ is bounded by a constant $C$ that is independent of $n$. It follows from the compact embedding of $C^{\alpha}(\bar{\Omega})$ into $C^{\beta}(\bar{\Omega})$ for $0<\beta<\alpha$ that there exists a subsequence $\left\{v_{n_{k}}\right\}$ of the sequence $\left\{v_{n}\right\}$ such that $v_{n_{k}} \rightarrow \phi$ in $C^{\beta}(\bar{\Omega})$. Since $\left\|v_{n_{k}}\right\|_{C^{0}(\bar{\Omega})}=1$, we have that $\|\phi\|_{C^{0}(\bar{\Omega})}=1$. Hence, $\phi \not \equiv 0$. Observe that $v_{n_{k}}$ is a weak solution of the equation

$$
\left.\begin{array}{rl}
-\Delta v_{n_{k}}+v_{n_{k}}=\lambda_{n_{k}} m(x) v_{n_{k}}+\frac{f\left(\lambda, x, u_{n_{k}}\right)}{\left\|u_{n_{k}}\right\|_{C^{0}(\bar{\Omega})}} & \text { in } \Omega, \\
\frac{\partial v_{n_{k}}}{\partial \nu}=\lambda_{n_{k}} \rho(x) v_{n_{k}}+\frac{g\left(\lambda, x, u_{n_{k}}\right)}{\left\|u_{n_{k}}\right\|_{C^{0}(\bar{\Omega})}} & \text { on } \partial \Omega .
\end{array}\right\}
$$

From (2.19) it follows that

$$
\frac{f\left(\lambda, x, u_{n_{k}}\right)}{\left\|u_{n_{k}}\right\|_{C^{0}(\bar{\Omega})}} \rightarrow 0 \quad \text { in } L^{r}(\Omega)
$$

and

$$
\frac{g\left(\lambda, x, u_{n_{k}}\right)}{\left\|u_{n_{k}}\right\|_{C^{0}(\bar{\Omega})}} \rightarrow 0 \quad \text { in } L^{r^{\prime}}(\partial \Omega)
$$

as $n_{k} \rightarrow \infty$. 
Using the weak formulation of (3.1) and passing to the limit, and also taking into account the fact that $\lambda_{n} \rightarrow \mu$ with $\mu \in \mathbb{R}$ and $v_{n_{k}} \rightarrow \phi$, we obtain that $\phi$ is a weak solution of the equation

$$
\left.\begin{array}{rlrl}
-\Delta u+u & =\mu m(x) u & & \text { in } \Omega, \\
\frac{\partial u}{\partial \nu} & =\mu \rho(x) u & & \text { on } \partial \Omega .
\end{array}\right\}
$$

Since $\phi \not \equiv 0$, it follows that $\mu$ is a generalized eigenvalue and $\phi$ is its corresponding eigenfunction. The proof is complete.

Corollary 3.2. Assume that the hypotheses of proposition 3.1 are satisfied.

If $u_{n} \geqslant 0$ for all $n$, then $\lambda_{n} \rightarrow \mu_{1}$ and the sequence $u_{n} /\left\|u_{n}\right\|_{C^{0}(\bar{\Omega})} \rightarrow \phi_{1}$ in $C^{\beta}(\bar{\Omega})$, where $\phi_{1}>0$ is the normalized eigenfunction associated with the first generalized eigenvalue $\mu_{1}$.

Proof. From proposition 3.1, we get that any possible convergent subsequence $u_{n} /\left\|u_{n}\right\|_{C^{0}(\bar{\Omega})} \rightarrow \phi_{i}$ for some $i \in \mathbb{N}$ with $\left\|\phi_{i}\right\|=1$. Since $u_{n} \geqslant 0$, we have that $\phi_{i} \geqslant 0$. From [17], we know that $\mu_{1}$ is the only generalized eigenvalue that is simple, so its corresponding eigenfunction does not change sign. Therefore, $\phi_{i}=\phi_{1}$.

Now, we shall show that any generalized eigenvalue $\mu$ of odd multiplicity is a bifurcation point from infinity, that is, there exists a sequence $\left(\lambda_{n}, u_{n}\right) \in \mathbb{R} \times H^{1}(\Omega)$ of solutions of (1.1) such that $\lambda_{n} \rightarrow \mu$ and that $\left\|u_{n}\right\|_{C^{0}(\bar{\Omega})} \rightarrow \infty$.

THEOREM 3.3. Assume that the nonlinearities $f$ and $g$ satisfy the conditions (C1)(C3). If $\mu$ is a generalized eigenvalue of odd multiplicity, then the set of solutions of (1.1), which we denote by $\mathcal{T}$, possesses an unbounded component, $\mathcal{D}$, which meets $(\mu, \infty) \in \mathbb{R} \times C(\Omega)$. Moreover, if $\Lambda \subset \mathbb{R}$ is an interval such that $\Lambda \cap\left\{\mu_{i}\right\}_{i=1}^{\infty}=\{\mu\}$ and $\mathcal{M}=\Lambda \times\{u \in C(\bar{\Omega}): u$ is bounded away from 0$\}$, then either

(i) $\mathcal{D} \backslash \mathcal{M}$ is bounded in $\mathbb{R} \times C(\bar{\Omega})$, in which case $\mathcal{D} \backslash \mathcal{M}$ meets the set $\{(\lambda, 0): \lambda \in$ $\mathbb{R}\}$ at $\left(\lambda_{0}, 0\right)$ such that $f\left(\lambda_{0}, \cdot, 0\right)=g\left(\lambda_{0}, \cdot, 0\right)=0$, or

(ii) $\mathcal{D} \backslash \mathcal{M}$ is unbounded.

If (ii) holds and $\mathcal{D} \backslash \mathcal{M}$ has a bounded projection on $\mathbb{R}$, then $\mathcal{D} \backslash \mathcal{M}$ meets $(\hat{\mu}, \infty)$, where $\mu \neq \hat{\mu} \in\left\{\mu_{i}\right\}_{i}$; that is, $\mathcal{D} \backslash \mathcal{M}$ meets another bifurcation point from infinity.

Proof. From (2.17) and (2.18), we see that (1.1) is equivalent to the fixed-point problem

$$
(u, \Gamma u)=\lambda S_{0} \circ \Theta(u, \Gamma u)+S_{0}(f(\lambda, \cdot, u), g(\lambda, \cdot \Gamma u)),
$$

where $S_{0}$ is the solution operator of (2.2). In order to apply [21, theorem 1.6], we need to verify the following:

(a) $S_{0}(f(\lambda, \cdot, u), g(\lambda, \cdot, \Gamma u))=o\left(\|u\|_{C^{0}(\bar{\Omega})}\right)$ at $u \rightarrow \infty$ uniformly on bounded $\lambda$ intervals;

(b) the $\operatorname{map}(\lambda, u) \rightarrow\|u\|^{2} S_{0}\left(f\left(\lambda, \cdot, u /\|u\|^{2}\right), g\left(\lambda, \cdot, \Gamma u /\|u\|^{2}\right)\right)$, where $\|\cdot\|:=$ $\|\cdot\|_{C^{0}(\bar{\Omega})}$, is compact. 
To verify (a), we first observe that if $u \in C(\bar{\Omega})$, then, by lemma 2.7 , we have that $(f(\lambda, \cdot u), g(\lambda, \cdot, \Gamma u)) \in L^{r}(\Omega) \times L^{r^{\prime}}(\partial \Omega)$ with $r>\frac{1}{2} N$ and $r^{\prime}>N-1$. Therefore, it follows from lemmas 2.2 and 2.7 that

$$
\begin{aligned}
\frac{\left\|S_{0}(f(\lambda, \cdot, u), g(\lambda, \cdot, \Gamma u))\right\|}{\|u\|} & \leqslant \frac{C\|(f(\lambda, \cdot, u), g(\lambda, \cdot \Gamma u))\|_{L^{r}(\Omega) \times L^{r^{\prime}}(\partial \Omega)}}{\|u\|} \\
& \leqslant C\left(\varepsilon+\frac{C_{\varepsilon}}{\|u\|}\right) .
\end{aligned}
$$

Therefore, (3.4) yields $S_{0}(f(\lambda, \cdot, u), g(\lambda, \cdot, \Gamma u))=o\left(\|u\|_{C(\bar{\Omega})}\right)$ at $u \rightarrow \infty$ uniformly on bounded $\lambda$ intervals.

To verify (b), it suffices to show that $T\left(\Lambda, B_{\delta}\right)$ is relatively compact in $C(\bar{\Omega})$ for some $\delta>0$, where $B_{\delta}=\{u \in C(\bar{\Omega}):\|u\| \leqslant \delta\}$. Pick $\delta>0$ very small and let $u \in B_{\delta}$. Considering $w=u /\|u\|^{2}$, it follows that $\|w\| \geqslant 1 / \delta$. Setting $\varepsilon=1$ in (2.19), we have that

$$
\frac{\|(f(\lambda, \cdot, u), g(\lambda, \cdot \Gamma u))\|_{L^{r}(\Omega) \times L^{r^{\prime}}(\partial \Omega)}}{\|u\|} \leqslant C,
$$

where $C$ is a constant depending on $\lambda$ and $\delta$. Hence,

$$
\|u\|^{2}\|(f(\lambda, \cdot, u), g(\lambda, \cdot, \Gamma u))\|_{L^{r}(\Omega) \times L^{r^{\prime}}(\partial \Omega)} \leqslant C\|u\| \leqslant C \delta .
$$

Using the compactness on $S_{0}$ in lemma 2.2 , we get that

$$
T(\lambda, u)=\|u\|^{2} S_{0}\left(f\left(\lambda, \cdot, u /\|u\|^{2}\right), g\left(\lambda, \cdot, \Gamma u /\|u\|^{2}\right)\right)
$$

is compact.

In the next corollary, we obtain a stronger result in the case of the simple eigenvalue $\mu_{1}$. The result below implies the existence of branches of positive and negative solutions bifurcating from infinity.

COROLlary 3.4. Let $\mu_{1}$ be the simple first generalized eigenvalue. Then the unbounded component $\mathcal{D}$ can be decomposed into two subcontinua $\mathcal{D}^{+}$and $\mathcal{D}^{-}$and there exists a neighbourhood $\mathcal{O} \subset \mathcal{M}$ of $\left(\mu_{1}, \infty\right)$ such that $(\lambda, u) \in \mathcal{D}^{+}\left(\mathcal{D}^{-}\right) \cap \mathcal{O}$ and $(\lambda, u) \neq\left(\mu_{1}, \infty\right)$ implies $(\lambda, u)=\left(\lambda, \alpha \phi_{1}+v\right)$, where $\alpha>0(\alpha<0)$ and $\left|\lambda-\mu_{1}\right|=o(1),\|v\|=o(|\alpha|)$ at $|\alpha|=\infty$, and $\phi_{1}$ is the eigenfunction corresponding to the first eigenvalue $\mu_{1}$.

Proof. One can use similar arguments to those in [21, corollary 1.8].

\section{Subcritical and supercritical bifurcations from infinity}

In this section, we shall focus on the type of bifurcation that occurs at the bifurcation point by providing conditions in which we have a sub- or supercritical bifurcation. Consider the problem

$$
\left.\begin{array}{rl}
-\Delta u+u=\lambda m(x) u+f(\lambda, x, u) & \text { in } \Omega, \\
\frac{\partial u}{\partial \nu}=\lambda \rho(x) u+g(\lambda, x, u) & \text { on } \partial \Omega,
\end{array}\right\}
$$


where $\mu$ is a generalized eigenvalue, and $(m, \rho) \in C^{0}(\Omega) \times C^{0,1}(\partial \Omega)$. Observe that, since $(m, \rho) \in C^{0}(\Omega) \times C^{0,1}(\partial \Omega)$, we have that $\phi_{1}>0$ in $\bar{\Omega}$ (see, for example, [16]).

We begin by defining the sub- and supercritical bifurcations.

DeFinition 4.1. A bifurcation from infinity at $\mu_{i}$ is said to be subcritical (respectively, supercritical) if there exists a neighbourhood $V$ of $\left(\mu_{i}, \infty\right)$ such that every (non-trivial) solution $(\lambda, u) \in V$ of (4.1) satisfies $\lambda<\mu_{i}$ (respectively, $\lambda>\mu_{i}$ ).

Now, let us first analyse the behaviour of solutions when we know that the solution blows up in the $C^{0}(\bar{\Omega})$ norm. Consider a sequence $\lambda_{n}$ for which the corresponding solutions $u_{n}$ of (1.1) are such that $\lambda_{n} \rightarrow \mu_{1}$ and $\left\|u_{n}\right\|_{C^{0}(\bar{\Omega})} \rightarrow \infty$ as $n \rightarrow \infty$. Since, for each $n \in \mathbb{N}, u_{n}$ is a weak solution of (1.1), we have that

$$
\begin{aligned}
\left(\mu_{1}-\lambda_{n}\right)\left[\int_{\Omega} m(x) u_{n} \phi_{1}\right. & \left.+\int_{\partial \Omega} \rho(x) u_{n} \phi_{1}\right] \\
& =\int_{\Omega} f\left(\lambda_{n}, x, u_{n}\right) \phi_{1}+\int_{\partial \Omega} g\left(\lambda_{n}, x, u_{n}\right) \phi_{1} .
\end{aligned}
$$

Note that, near the bifurcation point of positive solutions, the sum of the two integrals on the left-hand side of (4.2) becomes positive. Indeed, let $v_{n}=u_{n} /\left\|u_{n}\right\|_{C^{0}(\bar{\Omega})}$. It follows from proposition 3.1 that $v_{n} \rightarrow \phi_{1}$.

Now,

$$
\int_{\Omega} m(x) u_{n} \phi_{1}+\int_{\partial \Omega} \rho(x) u_{n} \phi_{1}=\left\|u_{n}\right\|_{C^{0}(\bar{\Omega})}\left[\int_{\Omega} m(x) v_{n} \phi_{1}+\int_{\partial \Omega} \rho(x) v_{n} \phi_{1}\right] .
$$

Taking the limit as $n \rightarrow \infty$, we get that

$$
\int_{\Omega} m(x) v_{n} \phi_{1}+\int_{\partial \Omega} \rho(x) v_{n} \phi_{1} \rightarrow \int_{\Omega} m(x) \phi_{1}^{2}+\int_{\partial \Omega} \rho(x) \phi_{1}^{2}>0 .
$$

Note that the sum of the last two integrals is positive because of conditions on the weight functions $m$ and $\rho$ and the fact that the eigenfunction associated with the first eigenvalue $\mu_{1}, \phi_{1}>0$.

Therefore, the sign of $\mu_{1}-\lambda_{n}$ is dictated by the asymptotic behaviour of the nonlinearities $f$ and $g$. We have the following additional conditions on the nonlinearities $f$ and $g$.

(C4) There exists $\gamma \in \mathbb{R}$ such that

$$
\begin{array}{lll}
\bar{F}_{+}(x):=\limsup _{(s, \lambda) \rightarrow(+\infty, \mu)} \frac{f(\lambda, x, s)}{s^{\gamma}}, & \bar{G}_{+}(x):=\limsup _{(s, \lambda) \rightarrow(+\infty, \mu)} \frac{g(\lambda, x, s)}{s^{\gamma},} \\
\underline{F}_{+}(x):=\liminf _{(s, \lambda) \rightarrow(+\infty, \mu)} \frac{f(\lambda, x, s)}{s^{\gamma}}, & \underline{G}_{+}(x):=\limsup _{(s, \lambda) \rightarrow(+\infty, \mu)} \frac{g(\lambda, x, s)}{s^{\gamma},} \\
\bar{F}_{-}(x):=\limsup _{(s, \lambda) \rightarrow(-\infty, \mu)} \frac{f(\lambda, x, s)}{s^{\gamma}}, & \bar{G}_{-}(x):=\limsup _{(s, \lambda) \rightarrow(-\infty, \mu)} \frac{g(\lambda, x, s)}{s^{\gamma}}, \\
\underline{F}_{-}(x):=\limsup _{(s, \lambda) \rightarrow(-\infty, \mu)} \frac{f(\lambda, x, s)}{s^{\gamma}}, & \underline{G}_{-}(x):=\limsup _{(s, \lambda) \rightarrow(-\infty, \mu)} \frac{g(\lambda, x, s)}{s^{\gamma}},
\end{array}
$$

with $\underline{F}_{-}, \bar{F}_{-}, \underline{F}_{+}, \bar{F}_{+} \in L^{1}(\Omega)$ and $\underline{G}_{-}, \bar{G}_{-}, \underline{G}_{+}, \bar{G}_{+} \in L^{1}(\partial \Omega)$. 
REMARK 4.2 .

(i) Note that $\bar{F}_{ \pm}, \underline{F}_{ \pm}, \bar{G}_{ \pm}$and $\underline{G}_{ \pm}$also depend on $\gamma$ and the generalized eigenvalue $\mu$.

(ii) Since $f$ satisfies condition (C2), if $\gamma \geqslant 1$, we have that $\underline{F}_{ \pm}=\underline{G}_{ \pm}=0$.

THEOREM 4.3 (subcritical bifurcation from infinity at the first eigenvalue). Assume that conditions (C1)-(C3) hold and suppose that there exists $\gamma<1$ such that (C4) holds. If

$$
\int_{\Omega} \underline{F}_{+} \phi^{1+\gamma}+\int_{\partial \Omega} \underline{G}_{+} \phi^{1+\gamma}>0 \quad\left(\text { respectively }, \int_{\Omega} \bar{F}_{-} \phi^{1+\gamma}+\int_{\partial \Omega} \bar{G}_{-} \phi^{1+\gamma}<0\right),
$$

then the bifurcation from infinity of positive solutions (respectively, negative solutions) at $\mu_{1}$ is subcritical. Moreover, if

$$
\int_{\Omega} \bar{F}_{+} \phi^{1+\gamma}+\int_{\partial \Omega} \bar{G}_{+} \phi^{1+\gamma}<0 \quad\left(\text { respectively, } \int_{\Omega} \underline{F}-\phi^{1+\gamma}+\int_{\partial \Omega} \underline{G}_{-} \phi^{1+\gamma}>0\right),
$$

then the bifurcation from infinity of positive solutions (respectively, negative solutions) at $\mu_{1}$ is supercritical.

In order to prove this theorem, we need the following auxiliary result.

Lemma 4.4. Suppose that the nonlinearities satisfy conditions (C1)-(C3). Let $\lambda_{n}$ be a sequence such that $\lambda_{n} \rightarrow \mu_{1}$ as $n \rightarrow \infty$ and let the corresponding solutions $u_{n}$ of (1.1) be such that $\left\|u_{n}\right\|_{C(\bar{\Omega})} \rightarrow \infty$ as $n \rightarrow \infty$. Then

(i) if $u_{n}>0$, we have

$$
\begin{aligned}
\frac{\int_{\Omega} \underline{F}_{+} \phi^{1+\gamma}+\int_{\partial \Omega} \underline{G}_{+} \phi^{1+\gamma}}{\int_{\Omega} m(x) \phi^{2}+\int_{\partial \Omega} \rho(x) \phi^{2}} & \leqslant \liminf _{n \rightarrow \infty} \frac{\mu_{1}-\lambda_{n}}{\left\|u_{n}\right\|_{C(\bar{\Omega})}^{\gamma-1}} \\
& \leqslant \limsup _{n \rightarrow \infty} \frac{\mu_{1}-\lambda_{n}}{\left\|u_{n}\right\|_{C(\bar{\Omega})}^{\gamma-1}} \\
& \leqslant \frac{\int_{\Omega} \bar{F}_{+} \phi^{1+\gamma}+\int_{\partial \Omega} \bar{G}_{+} \phi^{1+\gamma}}{\int_{\Omega} m(x) \phi^{2}+\int_{\partial \Omega} \rho(x) \phi^{2}},
\end{aligned}
$$

(ii) if $u_{n}<0$, we have

$$
\begin{aligned}
\frac{-\left(\int_{\Omega} \bar{F}_{+} \phi^{1+\gamma}+\int_{\partial \Omega} \bar{G}_{+} \phi^{1+\gamma}\right)}{\int_{\Omega} m(x) \phi^{2}+\int_{\partial \Omega} \rho(x) \phi^{2}} & \leqslant \liminf _{n \rightarrow \infty} \frac{\mu_{1}-\lambda_{n}}{\left\|u_{n}\right\|_{C(\bar{\Omega})}^{\gamma-1}} \\
& \leqslant \limsup _{n \rightarrow \infty} \frac{\mu_{1}-\lambda_{n}}{\left\|u_{n}\right\|_{C(\bar{\Omega})}^{\gamma-1}} \\
& \leqslant \frac{-\left(\int_{\Omega} \underline{F}_{+} \phi^{1+\gamma}+\int_{\partial \Omega} \underline{G}_{+} \phi^{1+\gamma}\right)}{\int_{\Omega} m(x) \phi^{2}+\int_{\partial \Omega} \rho(x) \phi^{2}} .
\end{aligned}
$$


Proof. We shall just prove (i), as (ii) can be shown in a similar way. From proposition 3.1 , there exists a subsequence of $u_{n}$ (that we again denote by $u_{n}$ ) such that

$$
v_{n}=\frac{u_{n}}{\left\|u_{n}\right\|_{C^{0}(\bar{\Omega})}} \rightarrow \phi_{1} \quad \text { in } C^{\beta}(\bar{\Omega}) .
$$

It follows by the weak definition of $u_{n}$ that

$$
\left(\mu_{1}-\lambda_{n}\right)\left[\int_{\Omega} m(x) u_{n} \phi_{1}+\int_{\partial \Omega} \rho(x) u_{n} \phi_{1}\right]=\int_{\Omega} f\left(\lambda_{n}, x, u_{n}\right) \phi_{1}+\int_{\partial \Omega} g\left(\lambda_{n}, x, u_{n}\right) \phi_{1} .
$$

Note that

$$
\int_{\Omega} f\left(\lambda_{n}, x, u_{n}\right) \phi_{1}=\left\|u_{n}\right\|^{\gamma} \int_{\Omega} \frac{f\left(\lambda_{n}, x, u_{n}\right)}{u_{n}^{\gamma}}\left(\frac{u_{n}}{\left\|u_{n}\right\|}\right)^{\gamma} \phi_{1} .
$$

Using Fatou's lemma and the fact that $v_{n} \rightarrow \phi_{1}$ and $\phi_{1}>0$, we have that

$$
\begin{aligned}
\liminf _{n \rightarrow \infty} \int_{\Omega} \frac{f\left(\lambda_{n}, x, u_{n}\right)}{u_{n}^{\gamma}}\left(\frac{u_{n}}{\left\|u_{n}\right\|}\right)^{\gamma} \phi_{1} & \geqslant \int_{\Omega} \liminf _{n \rightarrow \infty}\left[\frac{f\left(\lambda_{n}, x, u_{n}\right)}{u_{n}^{\gamma}}\left(\frac{u_{n}}{\left\|u_{n}\right\|}\right)^{\gamma}\right] \phi_{1} \\
& \geqslant \int_{\Omega} \underline{F}_{+} \phi^{1+\gamma} .
\end{aligned}
$$

Similarly,

$$
\int_{\partial \Omega} g\left(\lambda_{n}, x, u_{n}\right) \phi_{1}=\left\|u_{n}\right\|^{\gamma} \int_{\partial \Omega} \frac{g\left(\lambda_{n}, x, u_{n}\right)}{u_{n}^{\gamma}}\left(\frac{u_{n}}{\left\|u_{n}\right\|}\right)^{\gamma} \phi_{1} .
$$

Hence,

$$
\liminf _{n \rightarrow \infty} \int_{\partial \Omega} \frac{g\left(\lambda_{n}, x, u_{n}\right)}{u_{n}^{\gamma}}\left(\frac{u_{n}}{\left\|u_{n}\right\|}\right)^{\gamma} \phi_{1} \geqslant \int_{\partial \Omega} \underline{G}_{+} \phi^{1+\gamma} .
$$

Dividing (4.8) by $\left\|u_{n}\right\|^{\gamma}$, taking the limit and thanks to (4.3), we obtain that

$$
\liminf _{n \rightarrow \infty} \frac{\mu_{1}-\lambda_{n}}{\left\|u_{n}\right\|^{\gamma-1}} \geqslant \frac{\int_{\Omega} \underline{F}_{+} \phi^{1+\gamma}+\int_{\partial \Omega} \underline{G}_{+} \phi^{1+\gamma}}{\int_{\Omega} m(x) \phi_{1}^{2}+\int_{\partial \Omega} \rho(x) \phi_{1}^{2}} .
$$

So, the first inequality in (4.6) is proved. The third inequality is proved similarly, and the second inequality follows from the properties of limits. The proof is complete.

Proof of theorem 4.3. From lemma 4.4 and (4.4) we have that $\mu_{1}>\lambda_{n}$ for every $n$. Similarly, lemma 4.4 and (4.5) imply that $\mu_{1}<\lambda_{n}$ for every $n$. The proof is complete.

\section{Resonance problem}

In this section we are concerned with the resonance problem

$$
\left.\begin{array}{rlrl}
-\Delta u+u & =\mu_{1} m(x) u+f(x, u) & & \text { in } \Omega, \\
\frac{\partial u}{\partial \nu} & =\mu_{1} \rho(x) u+g(x, u) & & \text { on } \partial \Omega,
\end{array}\right\}
$$


where $\mu_{1}$ is the first generalized eigenvalue. Consider the following one-parameter family of problems:

$$
\left.\begin{array}{rlrl}
-\Delta u+u & =\lambda m(x) u+f(x, u) & \text { in } \Omega, \\
\frac{\partial u}{\partial \nu} & =\lambda \rho(x) u+g(x, u) & & \text { on } \partial \Omega .
\end{array}\right\}
$$

As we shall see, the behaviour of the possible bifurcations from infinity at $\lambda=\mu_{1}$ in (5.2) determines the existence of a solution of (5.1). First, observe that every possible bifurcation from infinity at $\mu_{1}$ is subcritical (respectively, supercritical) if and only if there exists $\varepsilon>0$ and $M>0$ such that, for every solution $(\lambda, u)$ of (5.2), if $\lambda \in\left(\mu_{1}, \mu_{1}+\varepsilon\right)$, then $\|u\|_{C^{0}(\bar{\Omega})}<M$ (respectively, if $\lambda \in\left(\mu_{1}-\varepsilon, \mu_{1}\right)$, then $\left.\|u\|_{C^{0}(\bar{\Omega})}<M\right)$.

THEOREM 5.1 (existence for the resonance problem). Consider the nonlinear problem (5.2) and suppose that the hypotheses of theorem 4.3 are satisfied. Assume also that every possible bifurcation from infinity at $\mu_{1}$ in (5.2) is subcritical or every possible bifurcation from infinity at $\mu_{1}$ in (5.2) is supercritical. Then there exists at least one solution of (5.1).

Proof. We shall prove that there exist $\left(\lambda_{n}, u_{n}\right)$ solutions of problem (5.2) with $\lambda_{n} \rightarrow \mu_{1}$ such that $\left\|u_{n}\right\|_{C^{0}(\bar{\Omega})}<M$. This follows from the fact that any possible bifurcation from infinity must be either subcritical or supercritical. From theorem 2.1 we know that, for any small $\varepsilon>0$, (5.2) has at least one solution for all $\lambda \in(\sigma-\varepsilon, \sigma+\varepsilon) \backslash\left\{\mu_{1}\right\}$. Assume that every possible bifurcation from infinity at $\mu_{1}$ is subcritical. It then follows that there exists a constant $M$ such that, for any $\lambda_{n}>\mu_{1}$ with $\lambda_{n} \rightarrow \mu_{1},\left\|u_{n}\right\|_{C^{0}(\bar{\Omega})}<M$. It follows from theorem 2.4 that $u_{n}$ is uniformly bounded in $C^{\alpha}(\bar{\Omega})$. Since $C^{\alpha}(\bar{\Omega})$ is compactly embedded in $C^{\beta}(\bar{\Omega})$, there exists a subsequence of $\left\{u_{n}\right\}$ (that we again denote by $\left\{u_{n}\right\}$ ) such that $u_{n} \rightarrow u$ in $C^{\beta}(\bar{\Omega})$. Using similar arguments to those in the proof of proposition 3.1 , we get that $u$ is a solution for (5.2) with $\lambda=\mu_{1}$. This completes the proof.

Based on results in theorem 4.3, we can deduce some consequences of theorem 5.1.

COROLlary 5.2. Suppose that one of the following Landesman-Lazer-type conditions is satisfied:

$$
\begin{aligned}
\left.\int_{\Omega} \underline{F}_{+} \phi^{1+\gamma}+\int_{\partial \Omega} \underline{G}_{+} \phi^{1+\gamma}>0 \quad \text { (respectively, } \int_{\Omega} \bar{F}_{-} \phi^{1+\gamma}+\int_{\partial \Omega} \bar{G}_{-} \phi^{1+\gamma}<0\right), \\
\left.\int_{\Omega} \bar{F}_{+} \phi^{1+\gamma}+\int_{\partial \Omega} \bar{G}_{+} \phi^{1+\gamma}<0 \quad \text { (respectively, } \int_{\Omega} \underline{F}_{-} \phi^{1+\gamma}+\int_{\partial \Omega} \underline{G}_{-} \phi^{1+\gamma}>0\right) .
\end{aligned}
$$

Then there exists at least one solution to (5.1).

\section{Results of anti-maximum-principle type}

In this section, we show the anti-maximum principle for the following linear problem:

$$
\left.\begin{array}{rlrl}
-\Delta u+u & =\lambda m(x) u+f(x) & & \text { in } \Omega, \\
\frac{\partial u}{\partial \nu} & =\lambda \rho(x) u+g(x) & & \text { on } \partial \Omega .
\end{array}\right\}
$$


Clement and Peletier [10] and Arcoya and Gámez [4] proved the anti-maximum principle for elliptic problems with Dirichlet boundary condition, where the parameter $\lambda$ is only in the differential equation in the interior. Arrieta et al. [5] proved the anti-maximum principle for the inhomogeneous linear Steklov problem, that is, the parameter $\lambda$ is only on the boundary. Now, we shall prove the anti-maximum principle for problem (6.1), where the parameter $\lambda$ is in the differential equation both in the interior and on the boundary.

TheOREm 6.1. Let $(m, \rho) \in C^{0}(\Omega) \times C^{0,1}(\partial \Omega)$ and let $m$ and $\rho$ satisfy the following condition:

$$
m(x)>0 \text { a.e. on } \Omega \text { and } \rho(x)>0 \text { a.e. on } \partial \Omega \text {. }
$$

For every $(f, g) \in L^{r}(\Omega) \times L^{r^{\prime}}(\partial \Omega)$ with $r>N$ and $r>N-1$, there exists $\varepsilon=\varepsilon(f, g)$ such that

(i) if

$$
\int_{\Omega} f \phi_{1}+\int_{\partial \Omega} g \phi_{1}>0
$$

then every solution $(\lambda, u)$ of (6.1) satisfies

(a) $u>0$ in $\Omega$ if $\mu_{1}-\varepsilon<\lambda<\mu_{1}$,

(b) $u<0$ in $\Omega$ if $\mu_{1}<\lambda<\mu_{1}+\varepsilon$,

(ii) if

$$
\int_{\Omega} f \phi_{1}+\int_{\partial \Omega} g \phi_{1}=0
$$

then every solution $(\lambda, u)$ of (6.1) with $\lambda \neq \mu_{1}$ changes sign on $\partial \Omega$ and consequently in $\Omega$.

Proof. (i) The Fredholm alternative theorem implies that the linear problem (6.1) has no solutions for $\lambda=\mu_{1}$, and a unique solution if $\lambda$ is not an eigenvalue of (1.2). Moreover, from theorem $3.3 \lambda=\mu_{1}$ is a bifurcation point from infinity, and from theorem 4.3 the bifurcation from infinity of positive solutions is subcritical, that is, there exists $\varepsilon=\varepsilon(f, g)$ such that, for every solution $(\lambda, u)$ of (6.1) with $\lambda \rightarrow \mu_{1}$, $\|u\| \approx \infty$ and $u>0$, we have $\mu_{1}-\varepsilon<\lambda<\mu_{1}$. Furthermore, the bifurcation from infinity of negative solutions is supercritical, that is, there exists $\varepsilon=\varepsilon(f, g)$ such that, for every solution $(\lambda, u)$ of (6.1) with $\lambda \rightarrow \mu_{1},\|u\| \approx \infty$ and $u<0$, we have $\mu_{1}<\lambda<\mu_{1}+\varepsilon$.

(ii) We first observe that (6.2) implies that $m$ and $\rho$ do not vanish simultaneously, and that either $m$ or $\rho$ is positive on subset of positive measure. To prove (ii), it suffices to multiply (6.1) by the eigenfunction $\phi_{1}$ and integrate by parts to get

$$
\left(\lambda-\mu_{1}\right)\left(\int_{\Omega} m(x) u \phi_{1}+\int_{\partial \Omega} \rho(x) u \phi_{1}\right)=0 .
$$

Note that since the function $m$ is continuous and the function $\rho$ is Lipschitz we have that $\phi_{1}>0$ in $\bar{\Omega}$ (see, for example, [16]). Now, since $\phi_{1}>0$ in $\bar{\Omega}$ and condition (6.2) holds, we conclude that $u$ changes sign in $\Omega$. 


\section{Acknowledgements}

The authors thank the referee for their careful reading of the manuscript and useful suggestions. N.M. was partly supported by the AWM-NSF Mentoring Travel Grant 2015. R.P. was partly supported by grant nos MTM2012-31298, MTM2016-75465, MINECO, Spain and Grupo de Investigación CADEDIF, UCM.

\section{References}

1 R. Adams and J. Fournier. Sobolev spaces, 2nd edn (Academic Press, 2003).

2 H. Amann. Nonlinear elliptic equations with nonlinear boundary conditions. In New developments in differential equations, ed. W. Eckhaus. North-Holland Mathematics Studies, vol. 21, pp. 43-63 (Amsterdam: North-Holland, 1976).

3 H. Amann. Fixed point equations and nonlinear eigenvalue problems in ordered Banach spaces. SIAM Rev. 18 (1976), 620-709.

4 D. Arcoya and J. L. Gámez. Bifurcation theory and related problems: anti-maximum principle and resonance. Commun. PDEs 26 (2001), 1879-1911.

5 J. M. Arrieta, R. Pardo and A. Rodríguez-Bernal. Bifurcation and stability of equilibria with asymptotically linear boundary conditions at infinity. Proc. $R$. Soc. Edinb. A 137 (2007), 225-252.

6 J. M. Arrieta, R. Pardo and A. Rodríguez-Bernal. Equilibria and global dynamics of a problem with bifurcation from infinity. J. Diff. Eqns 246 (2009), 2055-2080.

7 J. M. Arrieta, R. Pardo and A. Rodríguez-Bernal. Infinite resonant solutions and turning points in a problem with unbounded bifurcation. Int. J. Bifur. Chaos 20 (2010), 2885-2896.

8 H. Brézis. Functional analysis, Sobolev spaces and partial differential equations (Springer, 2011).

9 A. Castro and R. Pardo. Resonant solutions and turning points in an elliptic problem with oscillatory boundary conditions. Pac. J. Math. 257 (2012), 75-90.

10 P. Clement and L. A. Peletier. An anti-maximum principle for second-order elliptic operators. J. Diff. Eqns 34 (1979), 218-229.

11 L. C. Evans. Partial differential equations. Graduate Studies in Mathematics, vol. 19 (Providence, RI: American Mathematical Society, 1998).

12 A. Friedman. Partial differential equations (New York: Holt, Rinehart and Winston, 1969).

13 A. Küfner, O. John and S. Fučik. Function spaces (Groningen: Noordhoff, 1977).

14 O. A. Ladyzhenskaya and N. Uraltseva. Linear and quasilinear elliptic equations (London: Academic, 1968).

15 E. M. Landesman and A. C. Lazer. Nonlinear perturbations of linear elliptic problems at resonance. J. Math. Mech. 19 (1970), 609-623.

16 G. M. Lieberman. Boundary regularity for solutions of degenerate elliptic equations. Nonlin. Analysis TMA 12 (1988), 1203-1219.

17 N. Mavinga. Generalized eigenproblem and nonlinear elliptic equations with nonlinear boundary conditions. Proc. R. Soc. Edinb. A 142 (2012), 137-153.

18 J. Nečas. Les méthodes directes en théorie des équations elliptiques (Paris: Masson, 1967).

19 R. Pardo. Bifurcation for an elliptic problem with nonlinear boundary conditions. Rev. Integr. Temas Mat. 30 (2012), 151-226.

20 P. Rabinowitz. Some global results for nonlinear eigenvalue problems. J. Funct. Analysis 7 (1971), 487-513.

21 P. Rabinowitz. On bifurcation from infinity. J. Diff. Eqns 14 (1973), 462-475. 
